\title{
Is virtual reality really effective in Parkinson's disease?
}

\author{
Realidade virtual realmente é efetivo na doença de Parkinson?
}

Johnnatas Mikael Lopes ${ }^{1}$

Reading the paper "Effect of virtual reality in Parkinson's disease: a prospective observational study"1 published in volume 76 , number 2, of this journal, it is important to emphasize that it is relevant to the physiotherapeutic practice. This publication has included innovation in physiotherapy prescription in recent years, not only in Parkinson's disease care but for other neurological ${ }^{2}$ and musculoskeletal diseases ${ }^{3}$ as well. However, some errors, which may compromise the applicability of the findings, are evident in the above-mentioned article.

First, the authors claim theirs to be a prospective observational, cohort design, in the title and method section. However, the study was designed as a before-and-after intervention type, in which there is no control group ${ }^{4}$. To be characterized as a prospective cohort study, the outcome variables are observed to verify their change/occurrence after follow-up without intervention. Therefore, this study is not an observational study.

It would have been possible to characterize their study as a retrospective cohort if the data collected were secondary, coming from individual clinical information system records, and if the authors had applied the virtual reality rehabilitation protocol before the research began. This would have considerably changed the conclusions regarding the evidence level. The correct classification is pertinent for future observational or intervention design meta-analysis studies.

The second set of errors refers to the data analysis. The authors use Spearman's correlation to observe the relationship between the performance in virtual reality games (independent variables) and variables of the Dizziness Handicap Inventory, Berg Balance Scale and SF-36 domains (outcome variables), before and after the intervention thus, reporting in the results that there was improvement in the outcomes.

However, the analysis is inadequate because, if there was any systematic bias in the measurement of variables before and after intervention, this may not have been detected and the capacity for cause-effect inference would be skewed.
For example, the Berg Balance Scale variable was correlated with the Soccer Heading score before intervention $(r=0.65, p=0.005)$. No correlation was found after intervention $(r=0.43, p=0.09)$. This type of analysis did not allow the authors to infer a main effect or effect interaction of the independent variable. Thus, a more appropriate analytical strategy would have been analysis of variance or its corresponding non-parametric, or even generalized estimation equations ${ }^{5}$, to really measure the effect of the intervention. Correlation analyses are not a good choice in studies with dependent data (longitudinal analysis).

In the before and after Dizziness Handicap Inventory and Berg Balance Scale analyses, shown in Figure 1, it is impossible for the reader to check the magnitude differences reported without mean and standard deviation estimates, because for a paired $t$ test, this estimate information is essential. Figure 1 seems to depict the amplitude of the data, from the lowest to the highest score, which has low statistical and clinical utility. Another aspect is the veracity of the null hypothesis probability reported due to the absence of the critical " $t$ " ratio. Therefore, it could not estimate the effect's measure as Cohen's d, which would have revealed the clinical usefulness.

The third problem is that Table 3 compared the performance in virtual reality games before and after the intervention period. The difference in scores can only indicate that the participants learned more in one game than another, but this did not necessarily improve balance and quality of life dimensions, mainly because the previous correlation analysis does not support this proxy.

Finally, I think that virtual reality training is a good choice in the therapeutic management of Parkinson's disease due its applicability in contextual simulation and visual cues for movement planning. Clarification of equivocal results help the interpretation, clinical use and the usefulness of findings in meta-analysis studies that consider the design and the measures of effect.

${ }^{1}$ Faculdade Maurício de Nassau, Curso de Fisioterapia, Natal RN, Brasil.

Correspondence:Johnnatas Mikael Lopes; Av. Roberto Freire, 1514, Campim Macio; 59080-400 Natal RN, Brasil; E-mail: johnnataslopes2@gmail.com Conflict of interest: There is no conflict of interest to declare.

Received 05 April 2018; Received in final form 02 May 2018; Accepted 2 May 2018. 


\section{References}

1. Severiano MIR, Zeigelboim BS, Teive HAG, Santos GJB, Fonseca VR. Effect of virtual reality in Parkinson's disease: a prospective observational study. Arq. Neuro-Psiquiatr. 2018;76(2):78-84. https://doi.org/10.1590/0004-282X20170195

2. LanghorneP, Bernhardt J, Kwakkel G, Infi R. Stroke rehabilitation. The Lancet. 2011;377(9778):1693-702. https://doi.org/10.1016/S0140-6736(11)60325-5
3. Heidi S. Motor rehabilitation using virtual reality.J Neuroeng Rehabil. 2004 Dec;1(1):10. https://doi.org/10.1186/1743-0003-1-10

4. Medronho RA, Block KV. Epidemiologia. 2a ed. São Paulo: Atheneu; 2009.

5. Guimarães LSP, Hirakata VN. Uso do modelo de equações de estimações generalizadas na análise de dados longitudinais. Revista HCPA. 2012:32(4):503-11. 